\title{
LVII. On the amplitude of vibrations maintained by forces of double frequency
}

\section{N.C. Krishnaiyar M.A.}

To cite this article: N.C. Krishnaiyar M.A. (1922) LVII. On the amplitude of vibrations maintained by forces of double frequency, Philosophical Magazine Series 6, 43:255, 503-510, DOI: $10.1080 / 14786442208633903$

To link to this article: http://dx.doi.org/10.1080/14786442208633903

册 Published online: 08 Apr 2009.

Submit your article to this journal $[\pi$

Q View related articles $\longleftarrow$ 
they touch one pair of opposite magnetic poles in the turning part which represents the Weber element, with the result that one of is axes becomes fixed and rotation can consequently occur only about that axis. The magnetic properties of the model system then resenible those of pyrrhotite, a crystal of which, as Weiss * showed, will take up magnetic induction readily in one plane, but not in the direction perpendicular to that plane. The model may also be adjusted to exhibit differences of magnetic quality along different axes in the plane of magnetization. Such differences were in fact observed in pyrrhotite by Weiss. He found that when a crystal was turned about its non-magnetic axis in a fixed field there were abrupt magnetic changes at intervals of $60^{\circ}$. This is just what a study of the model would lead one to expect, for the projecting magnets in the turuing element lie in planes $60^{\circ}$ apart round any one of its magnets taken as axis. Periodic variations along axes inclined at $120^{\circ}$ to one another in the plane of easy magnetization are consistent with cubic symmetry on the part of the iron atom : they follow directly from the assumed grouping of magnet poles at the corners of a cube. And they-will occur at intervals of $60^{\circ}$ if we ascribe the bexagonal structure of the crystal to twinning in successive layers.

If Hull's view of the structure of the iron atom be correct, it seems not improbable that the Weber element includes not only the duplet of electrons which he places near the nucleus, but also the innermost octet, the members of which are somewhat further away from the nucleus, leaving the other and more distant octets to constitule what I bave called the fixed elements.

LVII. On the Amplitude of Vibrations maintained by Forces of Thouble Frequency. By N. C. Krishnaiyak, M.A., Lecturer in Physics, University College, Rangoon $\dagger$.

I ORD RAYLEIGH $¥$ was the first to discuss the theory $\mathcal{L}$ of the vibration maintained by an influence whose frequency was double that of the vibration maintained. A well-known example of such maintenance is the longitudinal form of Melde's experiment. His differential equation took the form

$k$ and $\alpha$ being small quantities.

$$
\ddot{y}+k \dot{y}+\left(n^{2}-2 \alpha \sin 2 p t\right) y=0,
$$

* Weiss, Jour. de Phys. iv. p. 469 (1900).

$\dagger$ Communicated by the Author.

$\ddagger$ 'Theory of Sound,' vol. i. pp. 81-85. 
Assuming

$$
\begin{aligned}
y=\mathrm{A}_{1} \sin p t+\mathrm{B}_{1} \cos p t & +\mathrm{A}_{3} \sin 3 p t+\mathrm{B}_{3} \cos 3 p t \\
& +\mathrm{A}_{5} \sin 5 p t+\mathrm{B}_{5} \cos 5 p t+\ldots
\end{aligned}
$$

and equating to zero the coefficients of $\sin p t$ and $\cos p t$ in the relation obtained when this value of $y$ was substituted in the differential equation, he showed that $A_{3}$ and $B_{3}$ were of the order $a$ and therefore negligible reiatively to $A_{1}$ and $B_{1}$. Hence as a first approximation,

Therefore

$$
\begin{aligned}
& \mathrm{A}_{1}\left(n^{2}-p^{2}\right)-(k p+\alpha) \mathrm{B}_{1}=0, \\
& \mathrm{~B}_{1}\left(n^{2}-p^{2}\right)+(k p-\alpha) \mathrm{A}_{1}=0 .
\end{aligned}
$$

$$
\frac{\mathbf{B}_{1}}{\mathbf{A}_{1}}=\frac{n^{2}-p^{2}}{k p+\alpha}=\frac{\alpha-k p}{n^{2}-p^{2}}=\frac{\sqrt{\alpha-k p}}{\sqrt{\alpha+k p}}
$$

and $\quad\left(n^{2}-p^{2}\right)^{2}=\alpha^{2}-k^{2} y^{2}$.

According to this theory, the phase $\tan ^{-1} B_{1} / A_{1}$ is constant and independent of the amplitude maintained, and the amplitude $\sqrt{\mathrm{A}_{1}{ }^{2}+\mathrm{B}_{1}^{2}}$ is indeterminate. But it is known * experimentally that the phase of the vibration is not independent of the amplitude, and a study of the maintained amplitudes brings ont a number of results which the above theory does not indicate.

In the case of fork maintenance the vibration of the wire reacts on the vibration of the fork and alters the amplitude of the latter, and so a difficulty arises in interpreting the results observed. The author $\dagger$ found that when an alternating current of 50 cycles, and therefore of 100 heat cycles, was passed through a wire whose length and tension were adjusted to a frequency of 50 , the wire on account of the periodic thermal expansion vibrated with a large amplitude. The effect of the earth's magnetic field was found to be negligible, the experiment succeeding equally well in all positions of the wire including the position when the wire was along the direction of the earth's magnetic force. The periodic thermal expansion due to the alternating current heating being independent of the vibration of the wire, there is no reaction between the vibrating system and the

* C. V. Raman, Physical Review, December 1912, pp. 451-453; N. C. Krishnaiyar, l'hysical Review., December 1919, pp. 494-496: R. N.Ghosh, Proc. Indian Assoc. Cultivation of Science, vol. vi, pp. $75 \& 84$ (1920).

$\dagger$ N. C. Krishnaiyar, "On the maintenance of vibrations of wires by electric heating," Physical Review, n.s. vol. vi. no. 6. 
maintaining influence. There is no mechanical force acting at a node or any other point of the wire. Hence a strict comparison of the observed amplitudes with theory is possible.

It is the aim of this paper to develop the theory so as to explain the facts of observation regarding the amplitude maintained. The details of the experiment a re given in the paper cited above, and it will suffice to summarize below the main results. $a$ is the constant factor of the periodic maintaining influence and in the experiment, for the same wire, depends upon the current through the wire and can be kept constant for an indefinite length of time or can be altered at will. $n$ is the natural frequency of the vibrating wire, and can be altered by altering the tension or the length of the wire, It was found that the maintenance started when the tension was slightly greater than that necessary to make $n=p$, i. e. when $p^{2}-n^{2}$ was negative. Maintenance continued as tension diminished so as to make $n=p$ and $n$ less than $p$, i. e. as $p^{2}-n^{2}$ became zero and as $p^{2}-n^{2}$ increased positively.

Fig. 1.

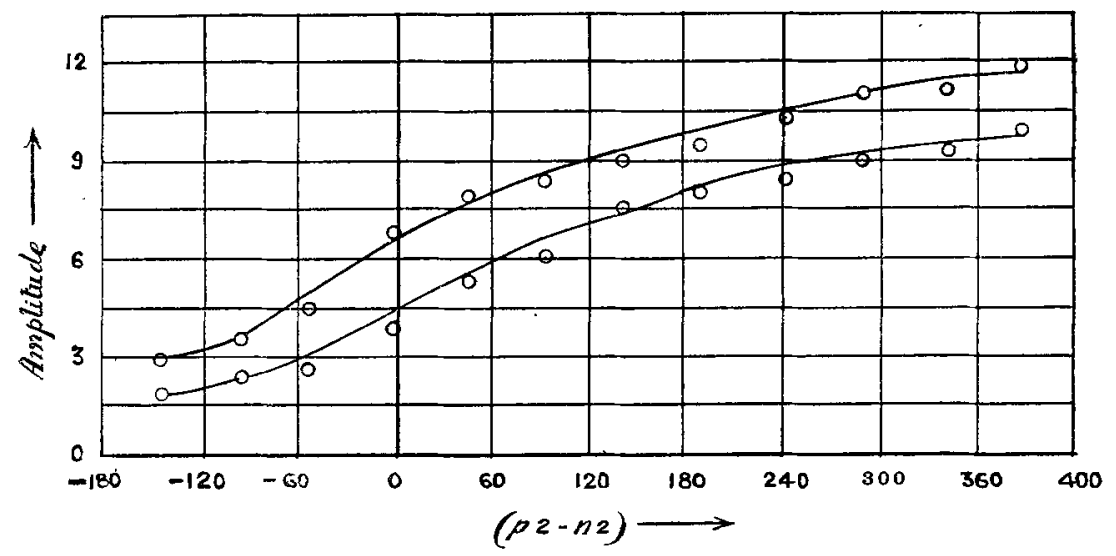

(1) When the observed amplitudes are plotted ${ }^{*}$ against the differences of the squares of the forced and the natural frequencies, there is no "peak" or maximum resonance as in the case of the ordinary forced oscillations, but only a continual increase in the amplitude from one end of the range where $p^{2}-n^{2}$ is negative to the other ond of the range where $p^{2}-n^{2}$ is positive.

(2) The amplitude increases to a maximum value after

* Figure 1 of the author's paper cited above. 


\section{Mr. N. C. Krishnaiyar on Amplitude of Vibrations}

which any diminution of tension brings about a sudden collapse of maintenance.

(3) The amplitude graph though parabolic in parts is not continually so ; the arc at one end produced parabolically does not coincide with the arc at the other end but is nearly: parallel.

(4) The maximum amplitude maintained increases if a increases in value.

(5) There is a lower limit to the value of $\alpha$ for maintenance to happen.

(6) There is an upper limit to the value of the excess tension, i.e. an epper limit to the magnitude of $p^{2}-n^{2}$ when $n>p$ if maintenance should happen. Beyond this, there is no vibration of the wire at all. The projection of the wire by a magic-lantern on a distant screen remains a sharp image.

(7) This upper limit to excess tension increases with increase of $\alpha$. In other words, when $\alpha$ is larger, maintenance starts with a larger excess tension.

Most of the observations detailed above have been confirmed by R. N. Ghosh * by an entirely different method of maintenance, viz. by means of an electric motor vibrator originally designed by J.A. Fleming and subsequently improved by C. V. Raman $\dagger$.

In the theory developed below, a term $\beta y^{2}$ is introduced in the part of the equation representing tension to denote the variation proved $\ddagger$ experimentally to occur in free oscillations of sensible amplitude. This variation is due to the second order differences in length between the equilibrium position and the displaced position of the wire and is proportional to the square of the displacement. The frictional force is put down as the sum of two terms, one proportional to the velocity and the other proportional to the square of the velocity. Ordinarily, as Sir G. G. Stokes has shown, the frictional force is proportional to the velocity when the velocity is small, and proportional to the square of the velocity when the velocity is relatively large. This apparent change of the law of friction with increase of velocity can be explained by the assumption that the frictional force always consists of two terms, the first term proportional to the velocity and the second term proportional to the square of the velocity, the coefficiont of velocity being relatively larger

* Proc. Indian Assoc. 1920, vol. vi.

+ Physical Review, November 1919.

+ C. V. Raman, "Photographs of vibration curves," Phil. Mag. May 1911 : Physical Review, December 1912. 
than the coefficient of the square of the velocity. When the velocity is small the first term will be the dominating term, and as the velocity increases, the second term will increase more rapidly than the first and will become ultimately the dominating quantity. Froude * has shown that the forced oscillations of a ship rolling among waves are due to a periodic forcing cause damped by resistance varying with both the first and the second powers of the angular velocity. Ronth $\dagger$ has shown analytically that the period of a pendulum in a very rare medium resisting partly as the velocity and partly as the square of the velocity, is constant throughout the motion and independent of the are. Parker Van Zandt $\ddagger$ has shown experimentally that the free vibrations of a system with a resisting torque due to both first and second power damping are isochronous. This law of frictional force when introduced into the differential equation brings ont all the above facts of experiment in double frequency maintenance.

The differential equation may be written as

$$
\ddot{y}+k^{\prime} \dot{y} \pm k \dot{y}^{2}+\left(n^{2}-2 \alpha \sin 2 p t+\beta y^{2}\right) y=0 .
$$

The sign \pm is introduced in the third term to indicate that that part of the frictional force changes sign as velocity changes sign.

Assume as before,

$$
\begin{gathered}
y=\mathrm{A}_{1} \sin p t+\mathrm{B}_{1} \cos p t+\mathrm{A}_{3} \sin 3 p t+\mathrm{B}_{3} \cos 3 p t \\
+\mathrm{A}_{5} \sin 5 p t+\mathrm{B}_{5} \cos 5 p t+\ldots,
\end{gathered}
$$

and consider in the limit all coefficients other than $A_{1}$ and $B_{1}$ to be negligible. Collect the coefficients of $\sin p t$ and $\cos p t$ with the aid of the following relations :

$$
\begin{aligned}
& y=\mathrm{A}_{1} \sin p t+\mathrm{B}_{1} \cos p t+\ldots \\
& \dot{y}=p\left(\mathrm{~A}_{1} \cos p t-\mathrm{B}_{1} \sin p t\right)+\ldots \\
& y \sin 2 p t=\frac{1}{2}\left(\mathrm{~A}_{1} \cos p t+\mathrm{B}_{1} \sin p t\right)+\ldots \\
& y^{3}=\frac{3}{4} \mathrm{~A}_{1}\left(\mathrm{~A}_{1}{ }^{2}+\mathrm{B}_{1}{ }^{2}\right) \sin p t+\frac{3}{4} \mathrm{~B}_{1}\left(\mathrm{~A}_{1}{ }^{2}+\mathrm{B}_{1}{ }^{2}\right) \cos p t+\ldots
\end{aligned}
$$

The above are easily obtained by differentiation and trigonometric transformation.

$$
\dot{y}^{2}=p^{2}\left(\mathrm{~A}_{1}^{2}+\mathrm{B}_{1}^{2}\right) \cos ^{2}\left(p t+\tan ^{-1} \mathrm{~B}_{1} / \mathbf{A}_{1}\right) \text {. }
$$

* Sir Phillip Watt's article on Shipbuilding in the Eneyclopadia Britannica.

† Routh's 'Advanced Rigid Dynamics,' Art. 364.

f J. Parker Van Zandt, Physical Review, November 1917. 
508 Mr. N. KC. Krisunaiyar on Amplitude of Librations

Expanding the even function $\cos ^{2}\left(p t+\tan ^{-1} \mathrm{~B}_{1} / \mathrm{A}_{1}\right)$ by Fourier's Series into a series of cosines of multiples of $\left(p t+\tan ^{-1} B_{1} / A_{1}\right)$ between the limits $-\frac{\pi}{2}$ and $\frac{\pi}{2}$, the term contsining $\cos \left(p t+\tan ^{-1} \mathrm{~B}_{1} / \mathrm{A}_{1}\right)$ is obtained as

$$
\frac{8}{3 \pi} p^{2}\left(\mathrm{~A}_{1}^{2}+\mathrm{B}_{1}^{2}\right) \cos \left(p t+\tan ^{-1} \mathrm{~B}_{1} / \mathrm{A}_{1}\right)
$$

and it can be written as

$$
\frac{8}{3 \pi} p^{2} \mathrm{~A}_{1} \sqrt{\mathrm{A}_{1}^{2}+\mathrm{B}_{1}^{2}} \cos p t-\frac{8}{3 \pi} p^{2} \mathrm{~B}_{1} \sqrt{{\mathrm{A}_{1}{ }^{2}+\mathrm{B}_{1}^{2}}_{2}} \sin p t .
$$

Equating the collected coefficients of $\sin p t$ and $\cos p t$ separately to zero, we obtain

and

$$
\begin{aligned}
\mathrm{A}_{1}\left\{n^{2}-p^{2}\right. & \left.+\frac{3}{4} \beta\left(\mathrm{A}_{1}{ }^{2}+\mathrm{B}_{1}{ }^{2}\right)\right\} \\
& =\mathrm{B}_{1}\left\{\alpha+\left[p k^{\prime}+\frac{8}{3} \frac{-2}{3} p^{2} k \sqrt{\mathrm{A}_{1}{ }^{2}+\mathrm{B}_{1}{ }^{2}}\right]\right\}
\end{aligned}
$$

$$
\begin{aligned}
& \mathrm{B}_{1}\left\{n^{2}-p^{2}+\frac{3}{4} \beta\left(\mathrm{A}_{1}^{2}+\mathrm{B}_{1}^{2}\right)\right\} \\
& =\mathrm{A}_{1}\left\{\alpha-\left[p k^{\prime} \pm \frac{8}{3 \pi} p^{2} k \sqrt{\mathrm{A}_{1}{ }^{2}+\mathrm{B}_{1}{ }^{2}}\right]\right\} .
\end{aligned}
$$

Therefore

$$
\left\{n^{2}-p^{2}+\frac{3}{4} \beta\left(\mathrm{A}_{1}{ }^{2}+\mathrm{B}_{1}{ }^{2}\right)\right\}^{2}=\alpha^{2}-p^{2}\left[k^{\prime} \pm \frac{8}{3 \pi} p k \sqrt{{\mathrm{A}_{1}{ }^{2}+\mathrm{B}_{1}{ }^{2}}^{2}}\right]^{2}
$$

If A stands for the amplitude of the maintained vibration,

$$
\begin{gathered}
\sqrt{\mathrm{A}_{1}{ }^{2}+\mathrm{B}_{1}{ }^{2}}=\mathrm{A} . \\
\therefore\left\{\frac{3}{4} \beta \mathrm{A}^{2}-\left(p^{2}-n^{2}\right)\right\}^{2}=\alpha^{2}-p^{2}\left[k^{\prime} \pm \frac{8}{3 \pi} p k \mathrm{~A}\right]^{2} .
\end{gathered}
$$

(1) The amplitude $A$ is not symmetrical with respect to $p^{2}-n^{2}$. So there will be no "peak" or maximum resonance. The last term on the right involving the squares and product of the small quantities $k$ and $k^{\prime}$ will be of small importance in the change of the value of $A$. So the relation between $A$ and $p^{2}-n^{2}$ will be nearly parabolic.

(2) Since the right-hand side $\alpha^{2}-p^{2}\left[k^{\prime} \pm \frac{8}{3 \pi} p k \mathrm{~A}\right]^{2}$ is equal to a square and therefore cannot be negative, the 
magnitude of A cannot be greater than $\frac{3 \pi}{8 p^{2} k}\left(\alpha-p k^{\prime}\right)$. Increase of $p^{2}-n^{2}$ above the value required to give this maximum value of A renders A imaginary. Hence a collapse of maintenance occurs.

(3) Substitute for $\frac{3 \pi}{8 p^{2} k}\left(\alpha-p k^{\prime}\right)$ the symbol $\mathrm{A}_{m}$ denoting maximum maintained amplitude.

Then

$$
\left\{\frac{3}{4} \beta \mathrm{A}^{2}-\left(p^{2}-n^{2}\right)\right\}^{2}=\alpha^{2}-\left\{p k^{\prime}+(\alpha-p k) \frac{\mathrm{A}}{\mathrm{A}_{m}}\right\}^{2} .
$$

At the excess-tension end, $A / A_{m}$ is very small and if neglected the graph reduces to the form

$$
\frac{3}{4} \beta \mathrm{A}^{2}=\sqrt{\alpha^{2}-p^{2} k^{\prime 2}}+\left(p^{2}-n^{2}\right) ;
$$

and at the defect-tension end, $\mathrm{A} / \mathrm{A}_{m}$ reduces to unity and the graph to the form

$$
\frac{3}{4} \beta A^{2}=p^{2}-n^{2} .
$$

The two limiting forms differ only by a constant. This shows that the shape of the graph at the two extremes is similar.

$$
\mathrm{A}_{m}=\frac{3 \pi}{8 p^{2} k}\left(\alpha-p k^{\prime}\right) \text {. }
$$

Therefore $A_{m}$ increases if $\alpha$ increases in value.

(5) The least value of $\alpha$ necessary for maintenance is that which makes the expression for the maximum maintained amplitade just equal to zero. Since $\mathrm{A}_{m}=\frac{3 \pi}{8 p^{2} k}\left(\alpha-p k^{\prime}\right)$, the lower limit of the value of $\alpha$ is $p k^{\prime}$. Or since $\alpha^{2}-p^{2}\left[k^{\prime} \pm 8 / 3 \pi p k \mathrm{~A}\right]^{2}$ is equal to a square, the least value of $\alpha$ is $p\left(k^{\prime} \pm 8 / 3 \pi p k \mathrm{~A}\right)$, and since there is no maintenance, $A=0$ and the least value of $\alpha$ is $p k^{\prime}$.

(6) At the excess-tension end, $\sqrt{\alpha^{2}-p^{2}} k^{\prime 2}+\left(p^{2}-n^{2}\right)$ is equal to $\frac{3}{4} \beta \mathrm{A}^{2}$ and $p^{2}-n^{2}$ is negative. So $p^{2}-n^{2}$ cannot be arithmetically greater than $\sqrt{x^{2}-p^{2} k^{\prime 2}}$. This sets an upper limit to the value of excess tension.

(7) This upper limit being $\sqrt{\alpha^{2}-p^{2} k^{\prime 2}}$. increases with increase in the value of $\alpha$.

If the frictional term involving the square of the velocity 


\section{Prof. C. V. Raman and Mr. V. S. Tamma on a}

be omitted, i.e. if $k$ be zero, the amplitude graph will become a strict parabola. There will be no upper limit to the value of the amplitude, and therefore the collapse of maintenance will not be explained. If the frictional term involving the first power of the velocity be omitted, i. e. if $k^{\prime}$ be zero, there will be no explanation for the fact that there is a lower limit to the value of $\alpha$ below which there is no maintenance. Facts (5) and (7) will be left unexplained. With the two frictional terms all the facts are indicated.

University College, Rangoon.

May 10, 1921.

I.VIII. On a New Optical Property of Biaxial Crystals. By (. V. Raman, M.A., Palit Professor of Physits in the Calcutta University, and V. S. TAMma, M.Sc., Lecturer in Physics, Meerut College, India *

\section{Introduction.}

1 FACT of singular interest and importance in the optics A of crystalline media which appears hitherto to have been overlooked is that a plate of a biaxial crystal bounded by parallel fices is capable of tocussing divergent rays proceeding from in distant light-source and forming real images of it. Very simple apparatus will suffice to observe this phenomenon. The incandescent filament of a tiny 2-volt lamp or an illuminated pin-hole serves as a suitable source of light. At some distance from it is placed a crystal of aragonite cut and polished with parallel-faces at right angles to the bisectrix of the acute angle between the optic axes. On suitably orienting the crystal and examining the pencil of light which has passed through it, a real erect unpoliarized image of the luminous filament may easily be picked up and traced continuously a way from the crystal for a considerable distance. There is no difficulty in receiving and observing the image directly on a plate of ground glass if desired.

The image is sharp and bright and practically achromatic if the object and the place of observation are both within a few centimetres of the crystal, one on each side. As either the object or the place of observation is drawn away from the crystal the image spreads out into a spectrum. Using monochromatic light, however, it can be seen that the image remains quite well defined and sufficiently bright to be

\footnotetext{
* Communicated by Prof. A. W. Porter, F.R.S.
} 